\title{
Photogeneration of charge carriers in photosensitive organic semiconductors
}

\author{
Yu.M. Barabash, M.A. Zabolotny*, N.I. Sokolov** \\ Institute of Physics, NAS of Ukraine, \\ 46, prospect Nauky, 03028 Kyiv, Ukraine \\ * T. Shevchenko University, 01033 Kyiv, vul. Volodymirs'ka, 64; \\ ** UKMA University, 04070 Kyiv, vul. Skovorody, 2
}

\begin{abstract}
Thermalization processes in photosensitive organic semiconductors are theoretically considered from the standpoint of their main parameters, namely: thermalization time and thermalization length. These are shown to be significantly dependent on the interaction of excitonic states with neighbours. The results of theoretical predictions are confirmed by the experimental ones obtained for a number of molecular semiconductors.
\end{abstract}

Keywords: organic molecular semiconductors, exciton, thermalization time, thermalization length.

Paper received 08.10.99; revised manuscript received 16.12.99; accepted for publication 17.12.99.

\section{Introduction}

The process of the electric charge carriers photogeneration in molecular semiconductors has been investigated [1,2], and it was found that this process was multi-stage. Two principal stages can be described. The first is the creation of the geminate electron-hole pairs bound by Coulomb field under the influence of the light quantum. The duration of this stage depends on the electron transition rate and usually lies in the range of $\left(10^{-12}-10^{-10}\right) \mathrm{s}$. During this time the neutral exciton states are formed and due to the autoionization turn into the pair ion - «hot» (nonequilibrum) charge carrier, which gives away its surplus energy in the process of unelastic collisions with other atoms and moves for the thermalization time $t_{T}$ to the thermalization length $r_{T}$. The thermalization process is said to be completed when the surplus energy of nonequilibrum charge carrier has decreased to the magnitude $\Delta \mathrm{W}$ when the carrier collision with its neighbours becomes elastic.

The experimental investigation of the thermalization process $[1,2]$ has shown that the thermalization length depends on the incident light frequency $(v)$, electric field strength $(E)$ and the molecular semiconductor temperature $(T)$. The typical dependences are shown in Fig. 1. A set of the models have been proposed [1-2] for the theoretical description of the thermalization process. The common shortcoming of these models is the necessity to make the additional assumptions about the behaviour of the model parameters in order to explain the experimental results. The variety of the approaches is caused by the lack of information on the proc- esses determining the formation and kinetics of an exciton state. In particular, the direction of the nonequilibrium charge carrier emission relatively to the light quantum momentum has not been clearly determined. If during the lifetime of an exciton state no significant change of its momentum occurs due to interactions with surroundings, the charge carriers will be emitted perpendicularly to the direction of the incident light quantum. Otherwise the direction of velocity of a charge carrier is not correlated with the direction of the momentum of light quantum. The suggested model allows to answer the question using experimentally obtained dependence $r_{T}(E)$.

The model of thermalization of nonequilibrium charge carriers is based on the following assumptions: 1) centres of photoluminescence are mutually independent; 2) a nonequilibrium charge carrier can be considered as a particle in thermal equilibrium with the surrounding; 3 ) the motion of the nonequlibrium charge carrier is determined by external electric field, the velocity of the nonequilibrium electric charge produced in the decay of exciton state and interaction of the nonequlibrium charge carrier with the medium. Coulomb interaction of geminal couple produced in the decay of exciton state is negligible. It is a most significant that the distance between the geminal charges would be small, i.e. when the velocity of the nonequilibrium charge carrier is large. It is assumed that interaction with the medium and inertia dominate for large nonequilibrium charge carrier velocities.

Taking into account the above assumptions and choosing $\mathrm{O} X$ axis along $\mathrm{E}$ the following equations can be obtained: 
Yu.M. Barabash et al.: Photogeneration of charge carriers in photosensitive...

$\frac{d V i(t)}{d t}+\gamma V_{i}(t)=\frac{F_{L}^{i}(t)}{m}+\frac{e E}{m} \delta_{i, x}$,

where $V_{i}(i=x, y, z)$ is a component of the velocity of nonequlibrium charge carrier, $\gamma$ is a friction constant, $m(e)$ is mass (charge) of the charge carrier, $F_{L}$ is a component of the Langivin force, which can be assumed as $\delta$-correlated:

$<F_{1}^{i}(t)>=0$,

$<F_{1}^{i}\left(L_{1}\right) F_{1}^{j}\left(t_{2}\right)>=D \delta\left(t_{1}-t_{2}\right) \delta_{i j}$

where $D$ is the intensity of Langivin source (here $<>$ denotes ensemble averaging). Initial conditions are taken as:

$\left.V_{i}(t)\right|_{t=0}=V_{i}(0) ; \quad V_{i}^{2}(0)=\frac{2}{m}(\eta v-A)$,

where $\mathrm{A}$ is the energy needed to create an exciton state. The solution of the problem is:

$$
\begin{aligned}
& V_{i}(t)=\frac{e E \delta_{i j}}{m \gamma}+\left(V_{i}(0)-\frac{e E \delta_{i j}}{m \gamma}\right) e^{-\gamma t}- \\
& -e^{-\gamma t} \int_{0}^{t} \frac{F_{L}^{2}\left(t^{\prime}\right)}{m} e^{\gamma t^{\prime}} d t^{\prime}
\end{aligned}
$$

From expressions (2), (3) it follows that

$$
\lim _{t \rightarrow \infty}<V_{i}^{2}(t)>=\left(\frac{e E}{m \gamma}\right)^{2}+\frac{3 D}{2 m^{2} \gamma} \text {. }
$$

The mean energy of a charge carrier in limit $t \rightarrow \infty$ must equal

$$
<E>=\frac{3}{2} K_{B} T+\frac{m}{2}\left(\frac{e E}{m \gamma}\right)^{2} \text {. }
$$

Comparing equations (5) and (6) one can found that

$D=2 m \gamma K_{B} T$.

Therefore

$$
\begin{aligned}
& <\frac{m V_{i}^{2}(t)}{2}>=\frac{m}{2}\left[\left(\frac{e E}{m \gamma}\right)^{2}+2 \frac{e E}{m \gamma}\left(V_{x}(0)-\frac{e E}{m \gamma}\right) e^{-\gamma t}+\right. \\
& \left.+V_{i}^{2}(0) e^{-2 \gamma t}+\left(\frac{e E}{m \gamma}\right)^{2} e^{-2 \gamma t}\right]+\frac{3 K_{B} E}{2}\left[1-e^{-2 \gamma t}\right]
\end{aligned}
$$

As was defined above, the thermalization process is completed when

$$
<\frac{m V_{i}^{2}(t)}{2}>=\Delta W+\frac{3 K_{B} T}{2} .
$$

Comparison of (8) and (9) leads to the equation for $t_{T}$ :

$$
\begin{aligned}
& \frac{2 \Delta W}{m}=\left(\frac{e E}{m \gamma}\right)^{2}+2 \frac{e E}{m \gamma}\left(V_{x}(0)-\frac{e E}{m \gamma}\right) e^{-\gamma t_{T}}+ \\
& +V_{i}^{2}(0) e^{-2 \gamma t_{T}}+\left(\frac{e E}{m \gamma}\right)^{2} e^{-2 \gamma t_{T}}- \\
& -V_{x}(0) \frac{e E}{m \gamma} e^{-2 \gamma t_{T}}-\frac{3 K_{B} T}{m} e^{-2 \gamma_{T}}
\end{aligned}
$$

by use the expression

$<r_{T}^{2}>=\int_{0}^{t_{T}} d t_{1} \int_{0}^{t_{T}} d t_{2}<V_{i}\left(t_{1}\right) V_{i}\left(t_{2}\right)>$

and

$<V_{i}\left(t_{1}\right) V_{i}\left(t_{2}\right)>=\left[\frac{e E}{m \gamma}+\left(V_{x}(0)-\frac{e E}{m \gamma}\right) e^{-\gamma t_{1}}\right] \times$

$\times\left[\frac{e E}{m \gamma}+\left(V_{x}(0)-\frac{e E}{m \gamma}\right) e^{-\gamma_{2}}\right]+$

$+\left(V_{y}^{2}(0)+V_{z}^{2}(0)\right) e^{-\gamma_{1}-\gamma_{2}}+$

$+3 K_{B} T e^{-\gamma t_{1}-\gamma t_{2}}\left(e^{\gamma t_{1}}-1\right)$

it follows that

$<r_{T}^{2}>=\left(\frac{e E}{m \gamma}\right)^{2} t_{T}^{2}+\frac{2 e E}{m \gamma^{2}}\left(V_{x}(0)-\frac{e E}{m \gamma}\right) \times$

$\times t_{T}\left(1-e^{-\gamma t_{T}}\right)+\frac{1}{\gamma^{2}}\left(V_{x}(0)-\frac{e E}{m \gamma}\right)^{2} \times$

$\times\left(1-e^{-\gamma t_{T}}\right)^{2}+\frac{3 K_{B} T}{m}\left(\frac{2 t_{T}}{\gamma}-\frac{2}{\gamma^{2}}+\frac{2}{\gamma^{2}} e^{-\gamma t_{T}}\right)$

The expressions (10) and (13) may be considered in two cases

$V_{i}(0) \sqrt{\frac{m}{h v-A}}<<1$

$V_{i}(0) \sqrt{\frac{m}{h v-A}} \approx 1$.

Condition (14) corresponds to $\Delta p / p<<1$, while (15) corresponds to the violation of this, $\Delta p=p$ (photon)- $p$ (charge carrier).

It can be shown that for (14)

$t_{T} \cong \frac{1}{2 \gamma}\left[\ln \frac{h v-A}{\Delta W}+\frac{e^{2} E^{2}}{2 m \Delta W \gamma^{2}}-\frac{3 K_{B} T}{2(h v-A)}\right]$,

$<r_{t}^{2}>\cong \frac{e^{2} E^{2}}{4 m^{2} \gamma^{4}}\left[\ln \frac{h v-A}{\Delta W}+\frac{e^{2} E^{2}}{2 m \Delta W \gamma^{2}}-\right.$

$\left.\frac{3 K_{B} T}{2(h v-A)}\right]+\frac{3 K_{B} T}{m \gamma^{2}} \times$

$\times\left[\ln \frac{h v-A}{\Delta W}+\frac{e^{2} E^{2}}{2 m \Delta W \gamma^{2}}-\frac{3 K_{B} T}{2(h v-A)}\right]$

In the case of (15) one has 
Yu.M. Barabash et al.: Photogeneration of charge carriers in photosensitive...

$$
\begin{aligned}
& t_{T} \cong \frac{1}{\gamma}\left[\ln \left(\frac{e E}{\gamma \Delta W} \sqrt{\frac{2}{m}(h v-A)}\right)+\frac{e^{2} E^{2}}{2 m \gamma^{2}}\right], \\
& <r_{T}^{2}>\cong \frac{2}{m \gamma^{2}}(h v-A)+\frac{6 K_{B} T}{m \gamma^{2}} \times \\
& \times \frac{\ln ^{2}\left[\frac{e E}{m \gamma} \sqrt{\left.\frac{2}{m}(h v-A)\right]}\right.}{\frac{2 W_{0}}{m}-\left(\frac{e E}{m \gamma}\right)^{2}}
\end{aligned}
$$

Conditions $\frac{e E r_{T}}{h v-A}<<1, \frac{K_{B} T}{h v-A}<<1$ are used in the derivation of (16) through (19).

The results of the numerical analysis of expressions (17), (19) are presented in Fig. 1. It is clear that the results of experiments with pentacene agree with (17). This points to the realization of the condition $\Delta p / p<<1$ by thermalization of electric charge carriers. The results of experiments with $\mathrm{PCV}$, on the other hand obey expression (19). It is possible when $\Delta p / p \sim 1$, in the case of large change of momentum of exciton during its lifetime. (Tab.1)

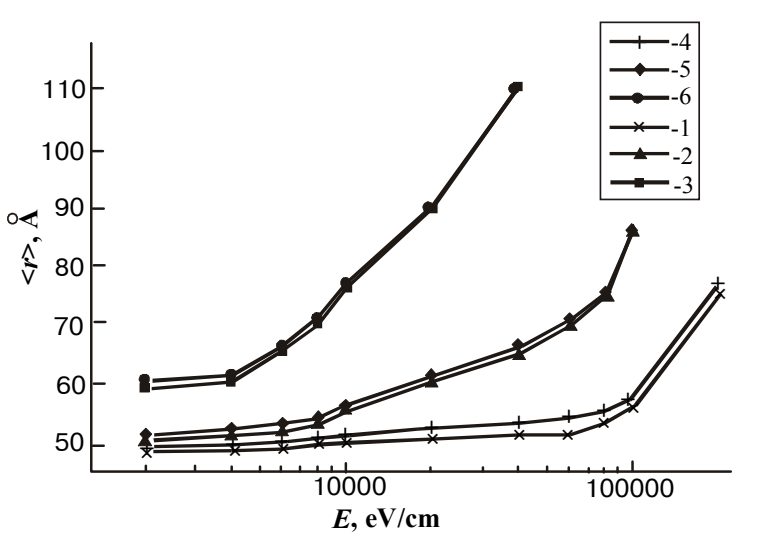

Fig.1. The experimental dependence of $r_{T}\langle E\rangle=\sqrt{\left\langle r_{T}^{2}(E)\right\rangle}$ for different quantum energies, $h v: 2,5$ (1), 2,6 (2), 2,8 eV (3). Curves (4), (5) and (6) are the theoretical dependences (in accordance with (17)).

\section{Conclusions}

A model of the thermalization of electric charge carriers is proposed to explain the thermalization process during the

\begin{tabular}{|c|c|c|c|c|c|c|c|c|}
\hline \multirow[t]{2}{*}{ FP } & \multirow{2}{*}{$\begin{array}{l}\text { Sensibili } \\
\text { zator }\end{array}$} & \multirow{2}{*}{$\begin{array}{l}\text { Quan } \\
\text { tity } \\
\text { mas.\% }\end{array}$} & \multicolumn{2}{|c|}{$S_{d v / v}=0.2 \mathrm{~m}^{2} / \mathrm{Dg}$} & \multicolumn{2}{|c|}{ Absorption, \% } & \multirow{2}{*}{$\begin{array}{l}\text { Change of } \eta \text { with } \\
\lambda \text { decrease from } \\
700 \text { to } 550 \mathrm{~nm} \\
\frac{S_{550}}{S_{700}}: \frac{\text { absorp } 550}{\text { absorp } 700}\end{array}$} & \multirow[t]{2}{*}{$E_{a}, \mathrm{eV}$} \\
\hline & & & $\lambda=700 \mathrm{~nm}$ & $\lambda=550 \mathrm{~nm}$ & $\lambda=700 \mathrm{~nm}$ & $\lambda=550 \mathrm{~nm}$ & & \\
\hline$\overline{\mathrm{PVC}}$ & TNF & 3.0 & 0.16 & 3.0 & 20 & 60 & $>6$ one & 2.05 \\
\hline$I_{d}=7,6$ & TENF & 5.0 & 1.2 & 8.0 & 40 & 75 & $>3,4$ one & 2.35 \\
\hline $\mathrm{eV}$ & TNFDCM & 3.0 & 3.0 & 6.0 & 60 & 85 & $>1,5$ one & 2.60 \\
\hline \multirow[t]{2}{*}{ PEPC } & $\mathrm{TNF}$ & 3.0 & 0.15 & 5.0 & 10 & 70 & 5.2 one & 2.05 \\
\hline & TENF & 5.0 & 1.3 & 7.0 & 46 & 78 & 3.4 one & 2.35 \\
\hline$I_{d}=7,6$ & Ud-DDFC & 4.0 & 3.0 & 8.0 & 55 & 70 & 2.1 one & 2.45 \\
\hline \multirow[t]{2}{*}{$\mathrm{eV}$} & TENFDCM & 3.0 & 3.5 & 7.0 & 60 & 80 & 1.6 one & 2.6 \\
\hline & TCXDN & 1.0 & 1.0 & 1.2 & 65 & 85 & 1 one & 2.7 \\
\hline \multirow[t]{2}{*}{ PAGE } & TNF & 4.3 & 0.4 & 5.0 & 10 & 75 & 2 one & 2.05 \\
\hline & TENF & 4 & 1.0 & 5.2 & 60 & 80 & 4 one & 2.35 \\
\hline$I_{d}=7,4$ & TNFDCM & 3.6 & 2.0 & 2.0 & 70 & 75 & 1 one & 2.60 \\
\hline $\mathrm{eV}$ & TCXDN & 1.0 & 0.8 & 0.8 & 50 & 70 & 0.9 one & 2.70 \\
\hline
\end{tabular}
decay in the external electric field of an exciton state, produced by light absorption in media with low charge mobility

Table1. The quantum yields of charge generation in photosensitive carbazol-containing materials. 
Yu.M. Barabash et al.: Photogeneration of charge carriers in photosensitive...

level. The model explains without introduction of extra model parameters temperature, field and energy dependence of the thermalization length. The character of the temperature dependence of the thermalization length depends significantly on the interaction of an exciton state with neighbours. In the case of weak interaction when momentum exchange between neighbours is insignificant, $r_{T}$ decreases with the medium temperature linearly or almost linearly. When momentum exchange is strong (i.e. when there is no correlation between the momentum direction in the beginning and in the end of its lifetime) $r_{T}$ only weakly depends on temperature. The specific shape of the dependence is determined by the details of the excess energy return by nonequilibrium charge carriers to the surroundings. Thermalization length of the photogenerated charge carriers in the case of weak interaction of exciton states with neighbours increases faster with the electric field than for strong interaction. Increase in energy of the light quanta, creating the exciton states in molecular semiconductors leads to the increase in the thermalization length. In the case of weak interaction this increase is logarithmic, while for strong interaction it is linear.

It is our pleasure to thank Prof. E.L.Frankevich for discussion of the presented results.

\section{References}

1. M.Pope, Ch.E.Swenberg, Electronic processes in inorganic crystals. Clarendon Press. Oxford. 1982, $621 \mathrm{p}$.

2. M.A.Zabolotny, Phenomenological model of charge carriers thermalization in molecuiar semiconductors., Europhysics Confer. Electronic Processes in Organic Materials., Kiev 18-22 M., 1998 